ESJ Social Sciences

\title{
Usages des Reseaux Sociaux par des Etudiants du Departement de Socioanthropologie a l'Universite Alassane Ouattara en Periode de Crise Sanitaire COVID 19
}

\author{
Dr. Essiomle Yawa Ossi, \\ Ecole Normale Supérieure d'Abidjan, \\ Département des Sciences de l'Education, Cote d'Ivoire
}

Doi:10.19044/esj.2021.v17n38p141

Submitted: 15 October 2021

Accepted: 25 November 2021

Published: 30 November 2021
Copyright 2021 Author(s)

Under Creative Commons BY-NC-ND

4.0 OPEN ACCESS

Cite As:

Essiomle Y.O. (2021). Usages des Reseaux Sociaux par des Etudiants du Departement de Socioanthropologie a l'Universite Alassane Ouattara en Periode de Crise Sanitaire COVID 19. European Scientific Journal, ESJ, 17 (38), 141.

https://doi.org/10.19044/esj.2021.v17n38p141

\section{Résumé}

Face à la psychose liée à la COVID-19 dans le monde, les individus et les institutions ont mobilisé divers mécanismes de résilience. A l'Université Alassane Ouattara (UAO) durant le confinement, des innovations pédagogiques, à savoir les enseignements par visioconférences, des cours en ligne et à distance à travers les mails sont entrepris. Plusieurs plateformes et espaces d'échange entre enseignants et étudiants ainsi qu'entre pairs étudiants sont créés. Toutefois, peu d'études ont été conduites pour renseigner avec exactitude sur les usages réels de ces réseaux sociaux par les étudiants durant le temps du confinement. L'objectif de cette étude est de déterminer les différents usages que font les étudiants du département de socio-anthropologie de l'UAO des réseaux sociaux en période de COVID-19. L'étude porte sur trente (30) sujets choisis de façon empirique dans les différents groupes sociaux (Facebook, Instagram, Telegram, WhatsApp, etc.). L'ancrage théorique de cette étude est la théorie de l'action raisonnée de Fishbein et Ajzen (1975). L'étude est qualitative, notamment phénoménologique. L'observation, la recherche documentaire et l'entretien semi-directif ont été utilisés comme techniques de recueils des données. Selon les résultats le facteur principal de l'utilisation des réseaux sociaux est l'anxiété suivie des activités lucratives et des besoins d'étude. En ce qui concerne, l'ordre de 
préférence des réseaux sociaux les plus visités WhatsApp vient en tête, suivi de Facebook, ensuite YouTube et enfin d'Instagram.

Mots clés : Effets psychosociaux, réseaux sociaux, crise sanitaire, COVID19 , usages, mesures barrières

\title{
Use of Social Networks by Students from the Socioanthropology Department at Alassane Ouattara University During the COVID 19 Health Crisis
}

\author{
Dr. Essiomle Yawa Ossi, \\ Ecole Normale Supérieure d'Abidjan, \\ Département des Sciences de l'Education, Cote d'Ivoire
}

\begin{abstract}
Faced with the psychosis linked to COVID-19 around the world, individuals and institutions have mobilized various resilience mechanisms. At Alassane Ouattara University (UAO) during confinement, educational innovations, namely teaching by videoconferencing, online and distance courses through emails are being undertaken. Several platforms and spaces for exchange between teachers and students as well as student peers are created. However, few studies have been conducted to provide accurate information on the real uses of these social networks by students during the time of confinement. The objective of this study is to determine the different uses that students of the socio-anthropology department of the UAO make of social networks during COVID-19. The study covers thirty (30) subjects chosen empirically from different social groups (Facebook, Instagram, Telegram, WhatsApp, etc.). The theoretical grounding of this study is the theory of reasoned action of Fishbein and Ajzen (1975). The study is qualitative, in particular phenomenological. Observation, desk research and semi-structured interviews were used as data collection techniques. According to the results the main factor in the use of social networks is anxiety followed by profitmaking activities and study needs. With regard to the order of preference of the most visited social networks WhatsApp comes first, followed by Facebook, then YouTube and finally Instagram.
\end{abstract}

Keywords: Psychosocial effects, social networks, health crisis, COVID-19, uses, barrier measures 


\section{Introduction}

Depuis le début des années 90, le monde a connu plusieurs crises sanitaires majeures dont celle du « sang contaminé » et celle de la « vache folle ». Ces crises se caractérisent par trois éléments essentiels: une origine très incertaine au début, un retentissement médiatique sans précédent ensuite et enfin une déstabilisation complète des systèmes institutionnels de gestion sanitaire. Le SRAS (Syndrome Respiratoire Aigu Sévère), la grippe aviaire et la grippe porcine constituant trois autres cas symptomatiques. La notion de sécurité sanitaire fait son apparition ainsi que les impératifs de santé publique qui sont au centre des missions de l'État. Pour les citoyens, les autorités doivent prendre leurs responsabilités, assurer leur protection contre les risques sanitaires et gérer la crise (Lajarge, Debiève et Nicollet (2013). Ces différentes pandémies ont mis en déroute le fonctionnement des sociétés, en l'occurrence l'éducation. En ce début d'année 2020, la crise sanitaire COVID-19 présentant les mêmes caractéristiques, que ces prédécesseurs a fait surface.

La COVID 19 a suscité un degré considérable de crainte, d'inquiétude et de préoccupation dans la population mondiale et chez certains groupes comme les personnes âgées, les prestataires de soins et les personnes souffrant d'affections préexistantes. Le principal impact psychologique à ce jour est un taux élevé de stress et d'anxiété. La prise de nouvelles mesures et l'émergence de nouveaux impacts - en particulier le confinement et ses effets sur les activités, les habitudes, les moyens de subsistance de nombreuses personnes les niveaux de solitude, de dépression, de consommation nocive d'alcool, d'usage de drogues, et de comportements auto-agressifs ou suicidaires ont également augmenté (World Health Organization [WHO], 2020 ; Inter Agency Standing Committee [IASC], 2020).

Face à la pandémie de COVID-19 l'école a été délocalisée à la maison. Ce changement de lieu d'apprentissage a causé des problèmes pédagogiques c'est-à-dire, des difficultés d'adaptation à la fois pour les élèves, les étudiants, les enseignants et même les parents. Les devoirs scolaires sont souvent source de tensions familiales et une tâche complexe à gérer pour les élèves et leurs parents. En outre, leur efficacité en termes d'apprentissage a été l'objet de nombreux débats (Glasman, 2004).

Cette pandémie qui touche la quasi-totalité des pays du globe a mis l'ensemble des services sanitaires à saturation, obligeant ainsi un confinement général de la population mondiale, ainsi que la fermeture de grandes institutions telles que les écoles, les universités grands foyers de contamination de la maladie. Cette situation qui bloque les interactions sociales entre les étudiants et paralyse le système éducatif, nécessite de recourir à d'autres moyens pour relancer le programme d'enseignement et maintenir les relations sociales entre les élèves, les étudiants et les enseignants. Pour sauver la situation, la solution immédiate et adaptée en ce temps de crise 
sanitaire est l'adoption de technologies éducatives, en d'autres termes l'implication des Technologies de l'Information et de la Communication pour l'Enseignement (TICE). Les établissements d'enseignement ont été contraints d'utiliser des systèmes d'apprentissage en ligne pour dispenser l'enseignement. Les cours en ligne, les webinaires et classes inversées sont mis en place pour maintenir les systèmes et services éducatifs qui sont aujourd'hui et seront à l'avenir, les moyens les plus efficaces pour le maintien des systèmes éducatifs. Les institutions de formation dans le monde entier auront recours aux technologies modernes et aux innovations pour offrir un enseignement de qualité non seulement à leurs citoyens mais aussi aux étudiants étrangers. Diverses plateformes de technologies éducatives entre autres Formative, Flipgrid, Insert Learning et Google Docs sont disponibles (Anti Partey, 2020). La Côte d'Ivoire n'est pas restée en marge de ce changement ; les autorités universitaires ont opté durant ce temps de la crise sanitaire du COVID 19 pour les plateformes de cours en ligne tels que Microsoft teams et Zoom, qui sont des applications de vidéos conférences qui devraient être optimisées et devenir une alternative à l'enseignement en présentiel. En outre, l'utilisation des réseaux sociaux pourraient être une alternative pour maintenir les contacts avec les enseignants et les étudiants ainsi qu'entre les étudiants eux-mêmes. Mais on constate que l'usage exact des réseaux sociaux par les étudiants pour les apprentissages reste un terrain à explorer surtout celui fait par les étudiants Ivoiriens.

Dans la littérature, il y a très peu d'études consacrées à la question de l'usage des réseaux sociaux en contexte de crises sanitaires. Pour Lévy (1997), les nouveaux moyens de communication permettent aux humains de mettre en commun leurs imaginations et leurs connaissances. Depuis près de 30 ans les recherches sur les technologies de l'information et de la communication pour l'enseignement sont restées très vastes (Moatti, 2010). Les travaux de Bruillard (2006) et de Fluckiger (2008) ont porté de façon spécifique sur les compétences mises en œuvre par les jeunes dans leurs usages des TICE ainsi que sur le lien avec les usages prescrits par l'école, le lycée et l'université. Aussi pour Delaunay-Téterel, 2010 et Metton-Gayon (2009), le cyber espace est un cadre de construction identitaire de la jeunesse. En outre, il favorise des acquissions de pratiques numériques personnelles (Fluckiger, 2008). Pour Pasquier (2005) et Dauphin (2012) les TICE et les réseaux sociaux sont perçus comme un cadre de socialisation des adolescents ; et d'émancipation à travers l'appartenance et l'attachement à un groupe ou une bande (Martin, 2004 ; Metton-Gayon, 2009). Au plan éducatif, l'usage des TICE dans l'éducation peut améliorer l'accès aux possibilités d'apprentissage et rehausser la qualité de l'éducation, à l'aide des méthodes pédagogiques de pointe afin d'accroître le rendement de l'apprentissage et de réformer les systèmes éducatifs et d'en améliorer la gestion (Institut de Statistique de 1'UNESCO [2010] ). En d'autres 
termes, c'est un outil permettant de faciliter l'apprentissage des élèves dans les différentes disciplines et niveaux d'études. Depover et Sterbelle (1997) préconisent l'implication de tous les acteurs susceptibles d'être touchés par cette innovation pour une intégration efficace de cette technologie dans les apprentissages scolaires.

L'usage des TICE en pédagogie universitaire a connu un accroissement rapide (Buckley, Pitt, Norton et Owens, 2010). Pour Margaryan, Nicol, Littlejohn et Trinder (2008) cité par Raby, Karsenti, Meunier \& Villeneuve (2011), l'accès à l'information est l'un des avantages essentiels des TICE pour l'étudiant universitaire. De plus, les TICE soutiennent l'autodétermination, l'exploration de l'identité, de même que la collaboration et le partage. L'étudiant a un accès facile, diversifié et libre partout où il se trouve (Rogers, 2001). Cet accès emmène l'étudiant à se responsabiliser et à s'engager face à son apprentissage et à sa réussite (Saunders et Klemming, 2003).

Le développement des technologies de l'information et de la communication (TIC) dans le milieu universitaire favorise non seulement de meilleures opportunités de diffusion des connaissances et d'accès à l'information mais aussi offre des possibilités de collaboration et de communication entre les enseignants et les étudiants (Oulmaati, Ezzahri et Samadi, 2017a). Pour Karsenti et Larose (2001), une motivation accrue chez les étudiants, une communication augmentée et améliorée, un accès plus important à l'information et aux connaissances, un enseignement plus efficace et plus individualisé et une plus grande autonomie des étudiants sont des avantages qui pourraient découler d'une intégration des TIC dans le processus d'apprentissage. Les travaux de recherche de Oulmaati, Ezzahri et Samadi (2017b) montrent que les outils de communication soutiennent de nouvelles formes de collaboration et de co-construction des connaissances. En outre, les activités en ligne et les outils technologiques favoriseraient également l'autorégulation et l'autonomie des étudiants (Monsakul, 2008). De plus, pour Rogers (2004) les TIC favorisent un apprentissage plus en profondeur [deeper learning] chez les étudiants universitaires.

Ainsi nous allons répondre dans cette étude à la question principale suivante :

De cette question principale découlent les questions spécifiques ci-après : Quels sont les réseaux sociaux les plus utilisés par les étudiants ? Quelles sont les raisons qui justifient le choix de ces réseaux sociaux ? Quelles est quantum horaire consacré par les étudiants à leurs apprentissages scolaires ?

L'objectif de cette étude est de déterminer les différents usages que font les étudiants du département socio-anthropologie de l'UAO des réseaux sociaux en période de COVID 19. 
La théorie de l'action raisonnée de Fishbein et Ajzen (1975) selon laquelle l'attitude d'une personne envers un comportement serait déterminée par ses croyances envers les conséquences de ce comportement multiplié par son évaluation de ces conséquences. Les croyances sont définies par la probabilité subjective de l'individu sur le fait qu'effectuer un comportement particulier va produire des résultats spécifiques. Ce modèle se base donc sur le postulat que les stimuli externes influencent les attitudes et cela en modifiant la structure des croyances de l'individu. Par ailleurs, l'intention d'effectuer un comportement est également déterminée par les normes subjectives qui sont elles-mêmes déterminées par les croyances normatives d'un individu et par sa motivation à se plier aux normes. Autrement dit, le choix des réseaux sociaux n'est pas fortuit et résulte d'une évaluation des avantages de tel réseau comparativement à tel autre réseau.

\section{METHODLOGIQUE \\ II.1. Terrain et population de l'étude}

Le terrain de cette étude est l'Université Alassane Ouattara (UAO) de Bouaké, ville située au centre de la Côte d'Ivoire et chef-lieu de la région du Gbêkè. Les bases de ce qui deviendra le 20 novembre 1995 «Université de Bouaké » ont été posées en 1992 dans le but de décongestionner l'Université Nationale de Côte d'Ivoire. Cette institution universitaire est installée sur deux sites à savoir le campus 1 qui abrite les UFR SJAG (Droit) et SED (Sciences économiques) et le campus 2 pour l'UFR Communication, Milieu et société (CMS). A ce jour, la population de cette université s'estime à plus de 20000 étudiants, 648 enseignants-chercheurs, 24 chercheurs et 338 personnels administratifs.

Dans le cadre de notre étude notre site de recherche se situera au campus 2 précisément au département de socio-anthropologie.

Le nombre total d'étudiants inscrits dans ce département est de 500 individus tous niveaux confondus. C'est au sein de cette population que nous avons choisi l'échantillon. Deux échantillonnages ont été utilisés de façon complémentaire. Dans un premier temps, il s'agit de l'échantillonnage à choix raisonné qui a consisté à sélectionner tous les étudiants consommateurs réguliers des réseaux sociaux en temps de COVID-19. Ensuite, l'on a procédé un échantillonnage stratifié non proportionnel ; étant donné que cette population se désagrège par niveau. Ainsi, 6 étudiants ont été choisis par niveau ce qui fait au total 30 individus.

\section{II.2. Techniques de recueil et d'analyse des données}

Dans le cadre de cette étude, l'observation, la recherche documentaire et de l'entretien semi-directif ont été utilisés. L'observation est une technique qui permet de recueillir des données verbales et surtout non verbales (Arborio, 
2007). Elle permet d'expliquer un phénomène à travers la description de comportements, de situations et de faits. Nous avons opté pour l'observation participante qui nous a permis de faire partie du contexte dans lequel nous avons mené notre étude; ainsi nous avons pu poser des questions aux personnes observées, qui sont les étudiants du département de socioanthropologie de l'UAO, et constater leurs habitudes d'utilisation des réseaux sociaux.

Quant à la recherche documentaire, elle correspond à l'ensemble d'" actions, méthodes et procédures ayant pour objet de retrouver dans des fonds documentaires les références des documents pertinents » (Association Française de Normalisation (AFNOR), 1993 : 99 cité par Dinet, Rouet et Passerault, 1998). Autrement dit, faire une recherche documentaire, c'est identifier et accéder à des ressources informationnelles qui ont déjà été traitées et éditées (Bibeau et Langlais, 1998). Dans le cadre de notre étude, la recherche documentaire nous a permis de répertorier et de recenser les écrits scientifiques, les archives ayant traités le sujet.

L'entretien, selon Guittet (2008), est une relation de face à face pour rechercher des informations, vérifier les faits, questionner, explorer, expliquer et commenter des idées. Dans le cadre de notre étude nous avons opté pour l'entretien semi-directif qui permet de centrer le discours des enquêtés autour de différents thèmes définis au préalable par le chercheur et consignés dans un guide d'entretien. Ce guide d'entretien est composé de quatre (4) thèmes principaux qui sont : les réseaux sociaux les plus utilisés avant le confinement et en période de confinement, les raisons du choix de ces réseaux sociaux, les facteurs du recours aux réseaux sociaux et le temps passé sur ces réseaux ainsi que les différentes activités sur les réseaux sociaux pendant et après le confinement. Les données recueillies ont fait l'objet d'une analyse qualitative, notamment phénoménologique.

\section{RESULTATS ET DISCUSSION \\ III.1. Résultats}

III.1.1 Réseaux sociaux les plus utilisés par les étudiants en période de la COVID 19

L'étude a révélé que les étudiants de l'UAO consultent plusieurs réseaux. Voici les réponses de certains participants par rapport aux réseaux sociaux les plus utilisés :

«Moi je vais plus sur WhatsApp parce que là si je vais c'est pour recueillir des informations qui peuvent me servir concernant mes études ... sur Facebook ce n'est pas évidant que j'aille là-bas comme ça» (participant \# 7 L2).

«Bon actuellement où je suis en train de te parler là c'est WhatsApp, voilà j'utilise plus WhatsApp et Facebook maintenant voilà après 
WhatsApp, il y a Facebook, il y a tweeter c'est généralement là c'est les trois là que j'utilise c'est maintenant là que je suis allé sur Telegram mais ça ne m'intéresse pas trop » (Participant \# 14 L3).

«Facebook, WhatsApp les deux... dès fois Tik-Tok...YouTube c'est quand on a les données pour souscrire les pass nuits pour télécharger les vidéos qu'on va là-bas » (Participant \# $16 \mathrm{~L} 3$ ).

«Bon moi généralement je suis entre Facebook et puis souvent Messenger ouais! Instagram je suis là-dessus mais où je travail le plus là c'est Facebook et puis WhatsApp... Snapchat j'ai peu de compte... Tweeter j'ai créé aussi mais généralement on dit ça c'est pour les boss, donc si tu n'as pas assez de connaissances qui sont à la hauteur de toi là-bas tu vois? Hors généralement nous nos amis c'est sur Facebook et WhatsApp » (Participant \# 8 L2).

«Si je veux faire un classement bah YouTube en première position; WhatsApp en deuxième position et Facebook en troisième position » (Participant \# 19 M1).

Le diagramme ci-dessous nous illustre les réseaux sociaux préférentiels des participants à cette enquête.

\section{Réseaux sociaux préférentiels des étudiants}

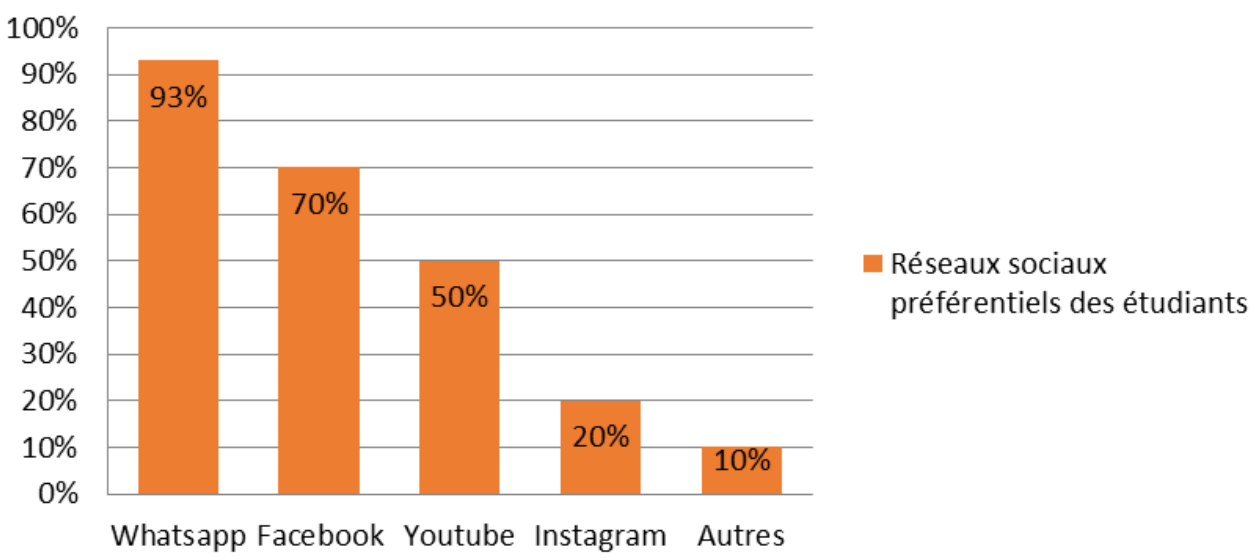

Figure 1 : Réseaux sociaux préférentiels des étudiants Source : données de l'enquête

Ce diagramme permet d'affirmer que WhatsApp (90\%), Facebook (70\%), et YouTube $(50 \%)$, autre à savoir Télégramme, Tweeter, Snapchat (10\%) sont les réseaux préférentiels des étudiants. 


\section{III.2. Raisons du choix de ces réseaux sociaux}

Plusieurs raisons sont évoquées pour donner les motifs d'augmentation du temps passé sur les réseaux sociaux : le contact des amis partis dans une autre ville ou dans une autre région, les études, le suivi de l'actualité sur la COVID-19 à cause de l'anxiété et le stress engendré par cette pandémie et l'ennui.

Voici quelques verbatims qui illustrent ces raisons :

"Tu t'ennui parce que même en venant... et puis à partir de $21 \mathrm{H}$ il y a couvre-feu, tu es à la maison tu n'as pas télé chez toi tu es seul là-bas tu vas causer avec qui, tu ne peux pas dormir tout le temps aussi ...donc normalement je passais beaucoup plus de temps sur les réseaux sociaux voilà » (Participant \# 15 L3).

«Oui oui c'est normal puisque pendant cette période là je ne pouvais pas sortir pour aller vaquer à mes occupations c'est le téléphone qui est là ...et puis je ne partais pas au travail comme ça donc forcément ça augmenté...et là je pouvais aller jusqu'à trois heures » (Participant \# 8 L2).

«Hum oui ça a changé un peu... vu la situation que ça concernait pas uniquement notre pays donc je regardais les vidéos qui ne parlait pas forcement de notre pays la cote d'ivoire donc vu cette situation c'est normal que je perds plus de temps sur YouTube pour pouvoir regarder les vidéos... pour les autres réseaux c'était pareil je mettais plus de temps » (Participant \# 19 M1).

\section{III.3. Facteurs du recours aux réseaux sociaux Facteurs liés à la fermeture de l'université}

La crise a entraîné entre autres mesure le recours aux réseaux sociaux chez plusieurs étudiants interrogés. Pour se prémunir des incidences psychoaffectives, de l'isolement occasionné par les fermetures des campus, les réseaux sociaux étaient de bon canaux d'échange et de distraction.

C'est ce que révèle la participante 1 de la Licence 1 :

"Oui oui ça eu un impact je me connectais plus que d'habitude, je causais plus avec mes amis puisque le campus était fermé on n'arrivait pas à se voir physiquement d'autres aussi avaient voyagé, donc c'est sur les réseaux sociaux qu'on communiquait plus » (participant \# 1 L1).

C'est dans cette optique que converge cet autre étudiant de la licence 2 quand il affirme :

« la communication se faisait via les réseaux sociaux comme je l'ai dit, mais plus je dirai avec WhatsApp puisque euh le site était fermé il y a des gens qui étaient rentrés en famille donc pour avoir des 
informations pour avoir de ces nouvelles comment est-ce que tu vas avec la maladie donc on faisait ça avec les réseaux sociaux» (participant \# 7 L2).

Les facteurs liés à la fermeture de l'Université Alassane Ouattara sont évoqués par les utilisateurs de réseaux sociaux de la première année à la cinquième. Toutefois, l'étude a révélé que certains étudiants ont, à contrario confié que la fermeture du campus a eu l'effet inverse sur leur usage des réseaux sociaux. Ceux-ci soutiennent qu'ils utilisaient moins les réseaux sociaux durant cette période. Ces participants ont déclaré avoir effectué des voyages dans des zones où la couverture réseau était très faible ; les empêchant ainsi d'être plus présent sur les réseaux sociaux. Voici les affirmations qui l'illustrent :

«la fermeture du campus? Bon c'est vrai ça eu un impact sur eux! Bon la communication entre mes condisciples puisque j'ai été au village où là-bas même le réseau pour appeler ce n'était pas facile d'avoir le réseau donc vraiment moi les réseaux sociaux je me connectais pas comme ça » (Participant \# 26 M2).

«euh depuis que je suis au campus ici pour me connecter j'utilisais notre wifi, notre wifi gratuit voilà donc quand il ont fermé les euh les la cité voilà je me suis retrouvé au village avec mes parents du coup je n'ai plus accès à mon wifi voilà donc au contraire ca diminuer ça n'a pas intensifier ça réduit le coût d'utilisation des réseaux sociaux... donc je ne pouvais pas acheter connexion chaque jour $500 f 200 f 300 f$ pour faire non je dois manger quand même ... voilà donc c'est difficile il faut se débrouiller pour manger et puis tu vas prendre ton argent pour faire ces genre de choses-là c'est pas c'est pas facile» (Participant \# 27 M2).

\section{Moyen de protection contre l'anxiété}

L'amplification des informations autour de la COVID-19 par les médias a plongé les étudiants dans une situation d'anxiété. C'est ce que révèle cet étudiant :

"Oui en tous cas au début là moi j'avais peur sincèrement à cause de tout ce que les gens disaient à la télévision. La progression rapide du virus et surtout le nombre de décès m'ont vraiment inquiété. Donc on partait sur les réseaux sociaux pour mieux s'informer afin de savoir comment éviter la maladie en appliquant les mesures barrières correctement $\gg$ (Participant \# $15 \mathrm{M} 1$ ).

«La peur du COVID-19 il faut dire que dans les débuts quand on parlait de signe de la maladie parce que on dit quand tu as le corps chaud. Une fois même j'avais le corps chaud parce que quand j'étais 
partir pour des entrainements avec les clubs là donc avec le déplacement Yopougon Abobo, quitter Yopougon Abobo aller-retour c'était fatiguant donc quand on a stoppé un le corps qui devais se reposer avant de reprendre j'avais commencé à sentir et le corps chaud et puis avec leur question de COVID qui commence par le corps chaud et autre là je me suis fait des idées ce qui faisais que je me voyais faire la maladie... voilà je me voyais faire la maladie donc du coup une peur en moi s'est installé, j'avais peur des conséquences... et puis un moment j'étais là un jour et je dis attend! Qu'est ce qui ne va pas? Attend je vais arrêter de penser à tout ça là. Et puis quand j'ai arrêté de penser j'ai repris mes esprits le lendemain, j'ai pris deux paracétamols étant donné que je chauffais c'est ma tête voilà j'ai pris deux paracétamols ... et puis voilà c'est parti »(Participant \# 28 M2).

Ainsi, les réseaux sociaux ont constitué pour d'autres un remède, un moyen d'évasion face aux effets psychosociaux de la COVID-19:

«Les réseaux sociaux nous permettait de penser à autre chose aussi parce que quand tu penses à coronavirus vrai vrai là ça faisait peur hein! on pensait que les gens allaient mourir du jour au lendemain donc euh voilà on partait plus là-bas pour se divertir pour penser à autre chose » (Participant \# 20 M1).

Enfin, les réseaux sociaux étaient un moyen pour accéder à l'information supposée vraie pour se donner bonne morale.

"Je partais sur Facebook YouTube ou bien Google pour m'informer de la situation, en tout cas je m'informais beaucoup sur COVID-19».

Pour cet autre enquêté, les contradictions autour des informations offraient aux consommateurs des réseaux sociaux l'opportunité de vérifier ses sources et distinguer les bonnes informations des fakes news :

«Ce que les gens disent sur les télévisions là les gens remettent ça sur les réseaux sociaux et puis les gens recommencent ça. Les différents commentaires sur les réseaux sociaux te permettent de te faire ton opinion afin de faire face à cette maladie »

\section{III.4. Temps consacré par les étudiants à leurs apprentissages avant la pandémie}

Le temps d'activité de chaque participant sur les réseaux sociaux par jour se situe dans les intervalles de [0-1[; [1-3[; [3-6[; [6-8]. En Licence 1, un participant sur six admet utiliser les réseaux sociaux moins d'une heure par jour, trois participants estiment utiliser les réseaux sociaux dans un intervalle de temps de trois à six heures et seulement deux participants utilisent les 
réseaux sociaux entre six et huit heures. En Licence 2, seulement un participant sur six a utilisé les réseaux sociaux moins d'une heure par jour, quatre participants sur six l'ont fait dans un intervalle de temps de trois à six heures et seulement un participant sur six entre six et huit heures. En Licence 3 , trois participants sur six admettent utiliser les réseaux sociaux moins d'une heure par jour, un participant sur six dans un intervalle de temps d'une à trois heures, un participant sur six entre trois et six heures et seulement un participant sur six entre six et huit heures. En Master 1, deux participants sur six ont utilisé les réseaux sociaux moins d'une heure par jour, trois participants sur six dans un intervalle de temps d'une à trois heures, un participant sur six entre six et huit heures. En Master 2, quatre participants sur six les ont utilisés moins d'une heure par jour, un participant sur six dans un intervalle de temps d'un à trois heures et seulement un participant sur six entre trois et six heures.

\section{Temps en moyenne passé sur les réseaux sociaux par les participants avant la pandemie}

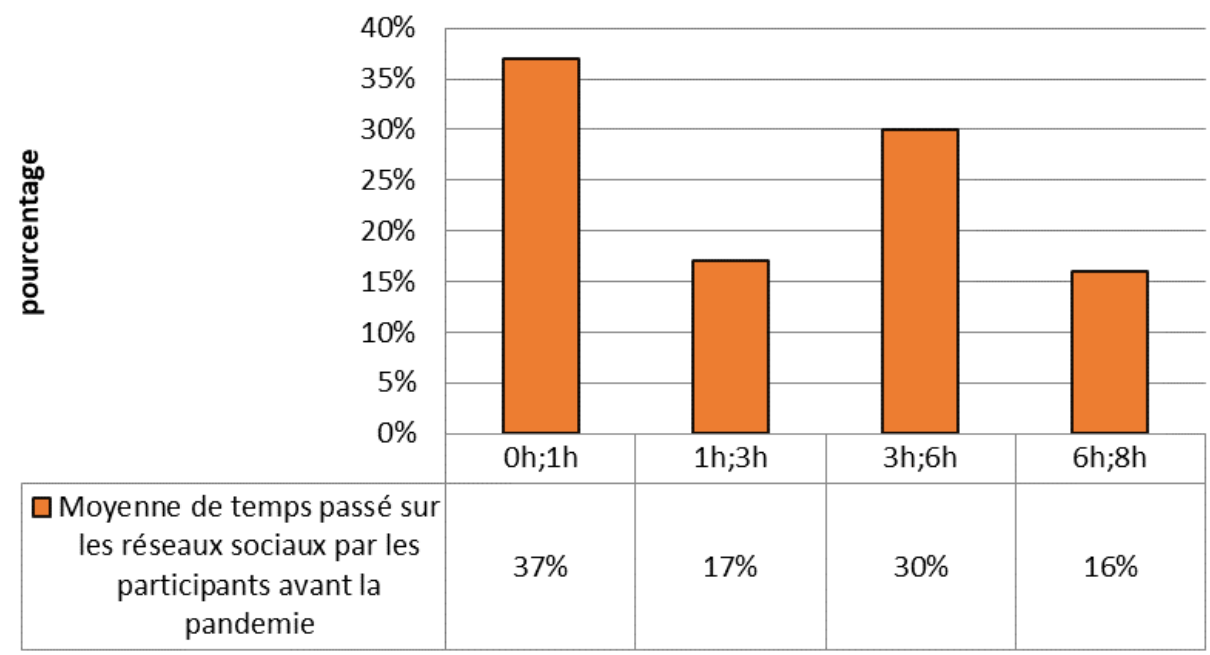

Figure 2 : Temps en moyenne passé sur les réseaux sociaux par les participants avant la pandémie Source : données de l'enquête

La figure 2 révèle que $37 \%$ des participants font moins d'une heure sur les réseaux sociaux soit 11 participants sur 30 ; environ $17 \%$ font entre une et trois c heures sur les réseaux sociaux soit un total de 5 participants ; $30 \%$ des participants passent en moyenne trois à six heures sur les réseaux sociaux ; $16 \%$ des participants sont présents sur les réseaux sociaux en moyenne six à huit heures de temps. 


\section{III.5. Différentes activités initiées par les étudiants sur les réseaux sociaux au début de la pandémie et à la reprise des cours}

Le tableau 1 en dessous indique les pourcentages des différentes activités initiées par les étudiants sur les réseaux sociaux selon deux (2) périodes bien définies qui sont au début de la crise sanitaire et à la reprise des cours.

Tableau 1 : Répartition des activités sur les réseaux sociaux par les étudiants selon la période

\begin{tabular}{c|c|c}
\hline PERIODE & $\begin{array}{c}\text { Temps T1 (Début de la } \\
\text { crise de la COVID-19) }\end{array}$ & $\begin{array}{c}\text { Temps T2 (Reprise des } \\
\text { cours) }\end{array}$ \\
\hline ACTIVITE & $17 \%$ & $40 \%$ \\
\hline ETUDE & $70 \%$ & $60 \%$ \\
\hline INFORMATION & $87 \%$ & $83 \%$ \\
\hline LOISIR ET DISTRACTION & $10 \%$ & $23 \%$ \\
\hline ACTIVITES & \multicolumn{2}{|c}{} \\
\hline
\end{tabular}

Source : données de l'enquête

Après analyse des données collectées sur le terrain nous avons pu établir une répartition d'activité sur les réseaux sociaux par les étudiants selon des périodes bien définies. Tout d'abord au début de la crise nous avons constaté que seulement $17 \%$ des étudiants interrogés, utilisaient les réseaux sociaux pour les études. $70 \%$ d'entre eux, se rendent sur les plateformes des réseaux sociaux pour s'informer. Et environ $87 \%$ des participants affirment utiliser les réseaux sociaux comme outil de loisir et de distraction. Ensuite lors de la reprise des cours nous avons remarqués que $40 \%$ des participants ont affirmés utiliser les réseaux sociaux pour les études, soit une augmentation de $23 \% .60 \%$ des participants ont utilisé les réseaux sociaux pour s'informer soit une baisse de $10 \% .83 \%$ d'entre eux ont déclaré les avoir utilisés pour le loisir et la distraction, soit une baisse de $4 \%$. En revanche, le volume horaire consacré à l'activité commerciale est passé du simple (10\%) au double (23\%). Il ressort de ce tableau que le temps d'utilisation le plus faible est celui consacré aux études. Les verbatims suivants viennent étayer les données du tableau :

"À vrai dire c'est en période de COVID-19 que j'ai commencé à vraiment m'intéresser à l'actualité ivoirienne sinon avant la période de COVID-19 bon c'était rare; c'était juste pour le divertissement...maintenant c'est en période de COVID que j'ai voulu voir comment ça se passais et tout donc j'ai commencé à $m$ 'abonner à certaine page... je causais beaucoup sur WhatsApp et je m'informais sur tweeter $\gg$ (Participant \# 2 L1).

«Il y avait des groupes qui ont été créé pour permettre aux étudiants de la Licence 1, bon avec la situation du corona bon! On va dire que 
on a été une génération sacrifiée et donc pour palier à ce problème, on a créé un groupe avec nos devancier appelé CONFINETYPE où là-bas ils répondaient à toute nos questions qu'on se posait ça c'était sur WhatsApp voilà et c'est pendant toute la période de confinement là qu'il le faisait mais une fois qu'ils ont arrêté le confinement il on aussi mit fin à ce groupe, si je peux dire ça comme ça... Ils se chargeaient de nous instruire, de nous enseigner puisqu'ont venais de rentrer et vous voyez qu'une semaine après il y eu le confinement donc pour ne pas qu'on rentre définitivement en brousse ils ont créé ce groupe » (Participant \# 2 L1)

«Aussi avec l'arrivé de la COVID -19 souvent aussi je partais là-bas pour avoir un peu d'information puisque sur Facebook puisque le ministère a sa page avec la venue du COVID -19. Donc souvent on va là-bas pour s'imprégner de la réalité de la situation en Côte d'Ivoire » (Participant \# 19 M1).

«Pendant la période de la COVID, je me connectais plus pour avoir des informations sur la question de la pandémie. Je me connectais pour avoir le minimum si je peux dire ça sur la maladie pour que demain si on me demande qu'est-ce que tu sais sur la COVID, je puisse un peu expliquer. Certes on donne des informations à la télé, mais ce n'est pas toutes les informations qu'on donne à la télé il y a des informations qu'on donne sur les réseaux sociaux...donc je me connectais plus sur les réseaux sociaux par rapport à cela» (Participant \# 7 L2).

"Il faut dire que moi j'ai appris, c'est-à-dire que pendant ce temps qu'il y a eu la pandémie j'ai vu des façons de pouvoir utiliser les réseaux sociaux pour commercialiser par exemple certains articles il $y$ avait certains commerçants aussi qui n'arrivaient pas à sortir pour vendre certains articles, j'ai vu qu'à travers les réseaux sociaux, j'arrivais facilement à faire la publicité la promotion de ces articles et donc moi c'est à partir de là que j'ai eu l'idée de rentrer aussi dans cette danse là pour aussi ,parce moi aussi je vends du Garba hein !... Je voulais un peu moderniser ça, rendre ça un peu industrielle je voulais à partir de mon WhatsApp faire des publications ... parce que j'ai vu que bon s'il arrive un moment où on n'arrive plus à sortir les réseaux sociaux sont une alternative pour pérenniser mon commerce » (Participant \# 22 M1).

\section{Discussion}

L'objectif de cette étude est de déterminer les différents usages que font les étudiants du département de socio-anthropologie de l'UAO des réseaux sociaux en période de COVID-19. Les principaux résultats de l'étude 
révèlent que les réseaux préférentiels des étudiants sont WhatsApp (90\%) suivi de Facebook (70\%), et YouTube $(50 \%)$ pour faire face aux effets psychosociaux de la crise sanitaire COVID-19. Ces résultats concordent avec les études de Nikiema (2013) qui énumèrent les usages les plus courants de l'Internet par le jeune public que sont la navigation sur les réseaux sociaux (Facebook, Twitter, Viadeo, LinkedIn...), les messageries instantanées, les blogs, les jeux en réseaux, etc. En ce qui concerne le temps utilisé sur les réseaux sociaux, il ressort de nos investigations que les étudiants consacrent peu de temps aux études qu'à d'autres activités comme la recherche des informations, les loisirs et la distraction ainsi que les activités commerciales. En outre, la majorité des participants ont déclarés passer plus de temps sur WhatsApp soit en moyenne trois à cinq heures par jour, parfois même toute la journée lorsque ceux-ci n'ont rien à faire, pour communiquer avec leurs amis. Sur Facebook leur passage était moins mais lorsqu'ils l'utilisaient, c'était pour la grande majorité soit pour s'informer ou se divertir; leur temps de présence ne dépassant pas généralement trente minutes. Contre toute attente, ces résultats montrent que YouTube n'enregistre que très peu de participants abonnés à ce réseau social mais dont les chiffres concernant la durée d'utilisation sont très élevés dépassant même parfois le temps d'utilisation de certains abonnés sur WhatsApp. Telegram fait partie des réseaux sociaux les moins utilisés par les étudiants interrogés car méconnus par ceux-ci. Il faut signaler que le temps accordé aux études sur les réseaux sociaux par les étudiants est faible que le temps consacré aux autres activités. Les étudiants y vont dans ce cadre des études de groupes sur WhatsApp ou pour demander de l'aide à leurs condisciples, mais également pour regarder des vidéos et des conférences sur YouTube. Aussi les étudiants passant plus de temps sur les réseaux sociaux sont en grande majorité ceux de la première année. Ces résultats coïncident avec ceux de Alhedaithy et Almobarraz, 2017 ; Hamdani, 2019 ; Manca et Ranieri, 2016 ; Roblyer, McDaniel, Webb, Herman et Witty, 2010 qui mettent en évidence le fait que les apprenants aient plus recours réseaux sociaux dans le processus E/A comparativement à leurs enseignants. Mais, ils sont distants de la position défendue par Delaunay-Téterel, 2010 et Metton-Gayon (2009) et Martin, (2004) qui affirment que les TIC et les réseaux sociaux sont un cadre de socialisation et d'émancipation à travers l'attachement à un groupe ou une bande donnée.

\section{Conclusion}

La crise sanitaire COVID-19 qu'a connu le monde et par ricochet, notre pays a eu une incidence majeure sur le déroulement du système éducatif. Cette situation a permis l'accroissement du recours aux TIC et aux réseaux sociaux comme alternative et outils pédagogiques dans l'apprentissage universitaire. L'objectif de cette recherche est principalement d'analyser la 
manière dont les étudiants utilisent les réseaux sociaux en période de COVID19 et plus spécifiquement identifier les différents réseaux sociaux utilisés par les étudiants ainsi que les raisons de leur utilisation et d'examiner les activités menées sur chaque type de réseau social. Les résultats de notre étude nous révèlent que les principaux réseaux sociaux utilisés par les étudiants sont : WhatsApp ; Facebook et Telegram ; aussi utilisent à des fins d'apprentissage, pour le divertissement et pour communiquer avec leurs camarades ; de plus, la durée sur les réseaux sociaux varie selon les réseaux et le type d'activité. Face à cette résurgence de l'usage des réseaux sociaux dans l'univers des étudiants, ne serait-il pas indispensable d'inclure dans leur maquette un module sur ces réseaux sociaux afin de les aider à mieux en tirer profit ?

\section{References:}

1. Alhedaithy, H. et Almobarraz, A. (2017). Adoption of Social Networks within Academic Context: A Diffusion of Innovation Approach. In: International Journal of Computer and Information Technology, 06 (03), (174-183).

2. Anti Partey, P. (2020). L'impact du COVID-19 et d'autres épidémies de maladies infectieuses sur les étudiants internationaux. Institute for Education Studies. Disponible en ligne à l'adresse https://www.globalpartnership.org/fr/blog/limpact-du-covid-19-etdautres-epidemies-de-maladies-infectieuses-sur-les-etudiants. Consulté le 20/05/2021 à 15h.

3. Arborio, A.-M. (2007). L'observation directe en sociologie : quelques réflexions méthodologiques à propos de travaux de recherches sur le terrain hospitalier. In: Recherche en soins infirmiers, 3 (90), (26 - 34).

4. Bibeau, R. et Langlais, P. (1998). La bibliothèque scolaire et Internet. Québec français, 110, (104-106).

5. Bruillard, E. (2006). Éditorial. In: Sciences et Technologies de l'Information et de la Communication pour l'Éducation et la Formation. Forum de Discussion en Education, 13, (235-254).

6. Buckley, C. A., Pitt, E., Norton, B. et Owens, T. (2010). Students approaches to study, conceptions of learning and judgments about the value of networked technologies. Active Learning in Higher Education, 11, (55-65).

7. Delaunay-Téterel, H. (2010). L'affichage public des amitiés Le blog au lycée. In: Ethnologie française 1 (40).

8. Depover, Ch., Strebelle, A. (1997). Un modèle et une stratégie d'intervention en matière d'introduction des TIC dans le processus éducatif. Luc-Olivier Pochon \& Alex Blanchet. L'ordinateur à l'école : de l'introduction à l'intégration. In : IRDP (73-98). 
9. Dauphin, F. (2012). «Culture et pratiques numériques juvéniles: Quels usages pour quelles compétences?». Questions Vives, 7 (17), |-1, (3752).

10. Dinet, J., Rouet, J-Fr. et Passerault, J-M. (1998). Les “ nouveaux outils " de recherche documentaire sont-ils compatibles avec les stratégies cognitives des élèves ?. Quatrième colloque "Hypermédias et Apprentissages". Poitiers (149-162).

11. Fishbein, M., \& Ajzen, I. (1975). Belief, Attitude, Intention, and Behavior: An Introduction to Theory and Research. Reading, MA: Addison-Wesley.

12. Fluckiger, C. (2008). «L'école à l'épreuve de la culture numérique des élèves». In: Revue française de pédagogie, 163, (51-61).

13. Glasman, D. (2004). Le travail des élèves pour l'école en dehors de l'école. Rapport établi à la demande du Haut conseil de l'évaluation de l'école (15).

14. Guittet, A. (2008). L'entretien: techniques et pratiques. $7^{\text {z̀me }}$ Edition. Paris : Armand Colin.

15. Hamdani, M. (2019). Technology Acceptance in the Use of Social Networks by Teachers and Employees of Education Offices in Ahwaz. In: The Turkish Online Journal of Educational Technology, 18 (1), (13).

16. Institut de Statistique de l'UNESCO (2010). Guide de mesure pour l'intégration des technologies de l'information et de la communication (TIC) en éducation. Montréal, Québec: ISU (Institut de Statistique de l'UNESCO).

17. Inter Agency Standing Committee [IASC]. (2020). Interim Briefing Note Addressing Mental Health and Psychosocial Aspects of COVID19 Outbreak. In: Reference Group on Mental Health and Psychosocial Support in Emergency Settings. IASC: Geneva.

18. Karsenti, T., et Larose, F. (2001). Les TIC au cœur des pédagogies universitaires : diversité des enjeux pédagogiques et administratifs. Québec : Presses de l'Université du Québec.

19. Lajarge, É., Debiève, H., Nicollet, Z. (2013). Santé publique: En 12 notions. Paris: Dunod.

20. Lévy, P. (1997). L'intelligence collective. Pour une anthropologie du cyberespace. Paris : La Découverte/Poche. Essais.

21. Manca, S. et Ranieri, M. (2016). Facebook and the others. Potentials and obstacles of Social Media for teaching in higher education. In: Computers \& Education, 95, (216-230).

https://doi.org/10.1016/j.compedu.2016.01.012, consulté le 8 juillet 2020 à $15 \mathrm{~h}$. 
22. Martin, O. (2004). L'internet des 10-20 ans. Une ressource pour une communication autonome. In: Lavoisier « réseaux », 1 (123), (25 - 58).

23. Metton- Gayon, C. (2009). Les adolescents, leur téléphone et Internet. «Tu viens sur MSN?» Paris L'Harmattan.

24. Moatti, D. (2010). Le numérique éducatif (1977-2009). 30 ans d'un imaginaire pédagogique officiel. Dijon: Éditions Universitaires de Dijon, coll. Sociétés.

25. Monsakul, J. (2008). A research synthesis of instructional technology in higher education. In: K. McFerrin et al. (dir.), Proceedings of Society for Information Technology \& Teacher Education (SITE) International Conference 2008 (2134-2139). Chesapeake, VA : Association for the Advancement of Computing in Education (AACE).

26. Nikiema, A. K. (2013). Utilisation des TIC : les dangers encourus par les jeunes apprenants, selon un spécialiste. Publié le samedi 23 novembre 2013. In : Le Quotidien. Disponible en ligne à l'adresse http://news.aouaga.com/h/17557.html. Consulté le 10/03/2021 à $10 \mathrm{H} 45$.

27. Oulmaati, K., Ezzahri, S., \& Samadi, K. (2017a). Impact des outils de communication intégrés à la plate-forme e-learning "minassati » sur l'activité d'apprentissage: Perceptions des étudiants inscrits en Histoire et Civilisation à l'université Abdelmalek Essaadi. EpiNet, (194).

28. Oulmaati, K., Ezzahri, S., et Samadi, K. (2017b). Impact des outils de communication intégrés à la plate-forme e-learning "minassati » sur l'activité d'apprentissage : Perceptions des étudiants inscrits en Histoire et Civilisation à l'université Abdelmalek Essaadi. EpiNet, (194).

29. Pasquier, D. (2005). Cultures lycéennes : la tyrannie de la majorité. Paris : Edition autrement - (mutations)

30. Raby, C., Karsenti, T., Meunier, H. \& Villeneuve, S. (2011). Usage des TIC en pédagogie universitaire : point de vue des étudiants. Revue internationale des technologies en pédagogie universitaire / International Journal of Technologies in Higher Education, 8 (3), (619).

31. Roblyer, M. D., McDaniel, M., Webb, M., Herman, J. et Witty, J. V. (2010). Findings on Facebook in higher education: A comparison of college faculty and student uses and perceptions of social networking sites. The Internet and Higher Education, 13 (3), (134-140). https://doi.org/10.1016/j.iheduc.2010.03.002, consulté le 8 juillet 2020 à $11 \mathrm{~h}$. 
32. Rogers, G. (2004). History, learning technology and student achievement: Making the difference? Active Learning in Higher Education, 5 (3), (232-247).

33. Rogers, P. L. (2001). Traditions to transformations: The forced evolution of higher education. AACE (Association for the Advancement of Computing in Education) Journal 9 (1), (47-60).

34. Saunders, G., \& Klemming, F. (2003). Integrating Technology into a Traditional Learning Environment Reasons for and Risks of Success. In: Active Learning in Higher Education 4, (74-86).

35. World Health Organization [WHO]. (2020). Mental health and psychosocial considerations during the COVID-19 outbreak 18 March 2020. New York: WHO. 